\title{
Implementación de una aplicación informativa cultural - educativa mediante Shiny para la provincia de
}

\section{Chimborazo}

\section{Implementation of an informative cultural - educational application through Shiny for the province of Chimborazo.}

Lourdes Emperatriz Paredes Castelo. ${ }^{1}$, Danny Israel Pilco Ninabanda. ${ }^{2}$, Beatriz del Carmen Manzo Moreira. ${ }^{3}$, \& Mariana Johanna Rodríguez Mayorga. ${ }^{4}$

Recibido: 10-05-2019 / Revisado: 15-06-2019 /Aceptado: 04-07-2019/ Publicado: 30-07-2019

\begin{abstract}
.
DOI: https://doi.org/10.33262/cienciadigital.v3i3.2.1.891

The objective of the research was to implement a technological platform through the use of free R-Shiny software to promote the tourist attractions of the ten cantons of the province of Chimborazo in Ecuador. The research was field, bibliographic, and a descriptive study was carried out with the help of a survey that determined the potential cultural themes to be promoted with priority: education, gastronomy, tourism, traditions and traditional dances. In addition, it was determined the acceptance that the computer application had and what were the expectations with the users. Through the statistical analysis of the information obtained with R-Shiny; the sustainability and sustainability of the project was encouraged and enhanced, seeking to increase the cultural and tourist market of the province of Chimborazo. Through the promotion, it contributed to the generation of employment and social, cultural and economic development. That is to say, that it had as a result, that citizens prefer the tourist and

${ }^{1}$ Escuela Superior Politécnica de Chimborazo, Facultad de Ciencias, Carrera de Ingeniería en Estadística Informática, lparedes@espoch.edu.ec

${ }^{2}$ Escuela Superior Politécnica de Chimborazo, Facultad de Ciencias, Carrera de Ingeniería en Estadística Informática, danny.pilcon@espoch.edu.ec

${ }^{3}$ Escuela Superior Politécnica de Chimborazo, Facultad de Ciencias, Carrera de Ingeniería en Estadística Informática, beatriz.manzo@espoch.edu.ec

${ }^{4}$ Escuela Superior Politécnica de Chimborazo, Facultad de Ciencias, Carrera de Ingeniería en Estadística Informática, mariana.rodriguez@espoch.edu.ec
\end{abstract}


gastronomic section; establishing that $78.2 \%$ of the people surveyed consider that the province lacks tourism impulse, which justifies the creation of the application and guarantees its sustainability.

Keywords: Webmapping, Emergency Management, Angular.js, Node.js, PostGIS

\section{Resumen}

El objetivo de la investigación, fue implementar una plataforma tecnológica a través del uso del software gratuito R-Shiny para promocionar los atractivos turísticos de los diez cantones de la provincia de Chimborazo en el Ecuador. La investigación fue de campo, bibliográfica, y se realizó un estudio descriptivo con la ayuda de una encuesta que determinó las potenciales temáticas culturales a ser promocionadas siendo prioritarias: la educación, gastronomía, turismo, tradiciones y danzas tradicionales. Además, se determinó la aceptación que tuvo la aplicación informática y cuáles fueron las expectativas con los usuarios. Mediante el análisis estadístico de la información obtenida con R-Shiny; se incentivó y potenció la sustentabilidad y sostenibilidad del proyecto, buscando incrementar el mercado cultural y turístico de la provincia de Chimborazo. A través de la promoción, se contribuyó a la generación de empleo y el desarrollo social, cultural y económico. Es decir, que se tuvo como resultado, que la ciudadanía prefiere la sección turística y gastronómica; estableciéndose que el 78,2\% de las personas encuestadas consideran que la provincia carece de impulso turístico, lo cual justifica la creación de la aplicación y se garantiza su sostenibilidad.

Palabras Claves: Shiny app, turismo, cultura, estadística

\section{Introducción.}

Según la Organización Mundial del Turismo (OMT, 1994), el turismo comprende las actividades que realizan las personas durante sus viajes y estancias en lugares distintos a su entorno habitual, por un periodo de tiempo consecutivo inferior a un año, con fines de ocio, por negocios y otros. Para otros especialistas, como Gurría (2004), Asanza, Matute (2006) y Ramírez, R. (2006), el turismo es un concepto impreciso, producto de su carácter interdisciplinario; sin embargo, admite múltiples definiciones y diversos matices, según el ámbito de trabajo y la época de que se formule el concepto. Pizam, Neumann y Reichel (1978) identificaron el término turistas satisfechos como un concepto nebuloso y poco investigado; además afirmaron que los investigadores y profesionales probablemente encontrarían este concepto muy difícil de definir.

Se puede tomar en cuenta el concepto de desarrollo de turismo sostenible, basándose en la necesidad de que la planificación, no se ciña a una disciplina determinada, sino que se enmarque en un trabajo global que considere al mismo tiempo los ámbitos económicos, sociocultural y ambiental, así como todas las prioridades de las partes afectadas por la actividad turística (residentes, visitantes, moradores del sector) (Crosby, 1996).

La actividad turística según su correcta planificación, debe ayudar a los sectores rurales a salir de su índice de pobreza; razón por la cual, incrementara su crecimiento económico. El 
turismo a nivel local o provincial en el Ecuador, es una actividad que contribuye al desarrollo económico de forma sustentable y sostenible; estudios realizados reflejan que en Ecuador entre 1990 y el 2009 la cantidad de visitantes extranjeros se elevó de 35.0000 a 90.0000 visitantes anuales, lo que evidencia un crecimiento notable en la actividad turística de nuestro país (Pizam, Neumann y Reichel, 1978).

Según lo expuesto por Cánoves, Herrera y Cuesta (2005), se debe considerar algunos aspectos para incrementar la demanda turística en cada región; como la planificación de buenas prácticas turísticas. En este ámbito, se consideran entre otros, el paisaje, la oportunidad de practicar deportes de invierno, la capacidad de acogida de los lugares, las edificaciones, el patrimonio arquitectónico, arqueológico, histórico o cultural, la proximidad a la franja marítima, y la accesibilidad del lugar.

El impacto de los valores culturales en el comportamiento del consumidor, han sido considerados en el mundo académico y empresarial, ya que está relacionado con la satisfacción del cliente y la percepción de la calidad del servicio recibido. Es así que, tomando en cuenta el término de satisfacción turística; se propuso crear una aplicación informática, en donde los turistas tengan información disponible en internet, que permita encontrar información referente a la cultura, gastronomía, danzas, lugares turísticos destacados. La provincia de Chimborazo de acuerdo con el último censo del 2010 posee una población de 458.581 habitantes distribuidos en sus diez cantones: Riobamba, Alausí, Chambo, Chunchi, Colta, Cumandá, Guano, Pallatanga y Penipe; los cuales poseen una cultura diversa: educación, gastronomía, turismo, tradiciones, danzas y patrimonio tangible e intangible en general.

Shiny es un paquete de $\mathrm{R}$, que permite construir aplicaciones web interactivas (Martín, Bustamante y Sánchez, 2019). Shiny muestran resultados dinámicos a través de la interfaz de usuario (UI); en donde se controla el diseño y aspecto de la aplicación (app), el servidor (server) se encarga de procesar e incorporar las instrucciones de entrada de datos con el uso del software gratuito $\mathrm{R}$, mediante el uso de funciones de paquetes que han sido instalados previamente y finalmente Shiny app crea el objeto de la aplicación. El uso de plataformas tecnológicas en la actualidad, se han convertido en uno de los métodos más usados para posicionar un proyecto, un emprendimiento o una marca en general.

Un estudio realizado por la consultora Flurry Analytics, evidenció que el tiempo que un usuario dedica al uso de aplicaciones específicas en internet es del 86\%; mientras que el 14\% restante solo usa un navegador, justificando así, la implementación de una aplicación específica con el uso del software libre R Shiny, la cual permite publicar en internet información relevante y referente a los atractivos turísticos de la provincia de Chimborazo, con el objetivo de alcanzar potenciales consumidores locales, nacionales e internacionales. Además, proporcionara información importante para las autoridades locales y nacionales, así como información de agentes económicos, para diseñar estrategias y procesos de planificación de destinos turísticos (Leon, 2018).

La justificación de este requerimiento de consumo turístico se evidencio a través de un estudio descriptivo, a través de encuestas dirigido a distintos segmentos de la ciudadanía de 
la provincia de Chimborazo, el cual determinó el grado de aceptación que tendría la aplicación denominada Nuka Kawsay (Mi cultura).

\section{Materiales y Métodos}

\section{Plataforma Tecnológica}

La creación de la aplicación informática cultural - educativa. se implementó utilizando dos scripts UI y un server, con el objetivo de mostrar resultados relevantes con el uso de la estadística y de sus herramientas tecnológicas, lo que permitió al usuario tener la oportunidad de interactuar y de conocer su uso y facilidades de navegación que posee el software.

La construcción de la aplicación informática "Ñuka Kausay", permitió desplegar información acerca de: gastronomía, cultura, lugares turísticos relevante, danzas tradicionales, información relacionada a los centros de educación importantes a nivel de país, tienda en línea de los diferentes productos que oferta cada cantón, como se puede observar en la Figura 1.

Figura 1. Aplicación Ñuka Kawsay

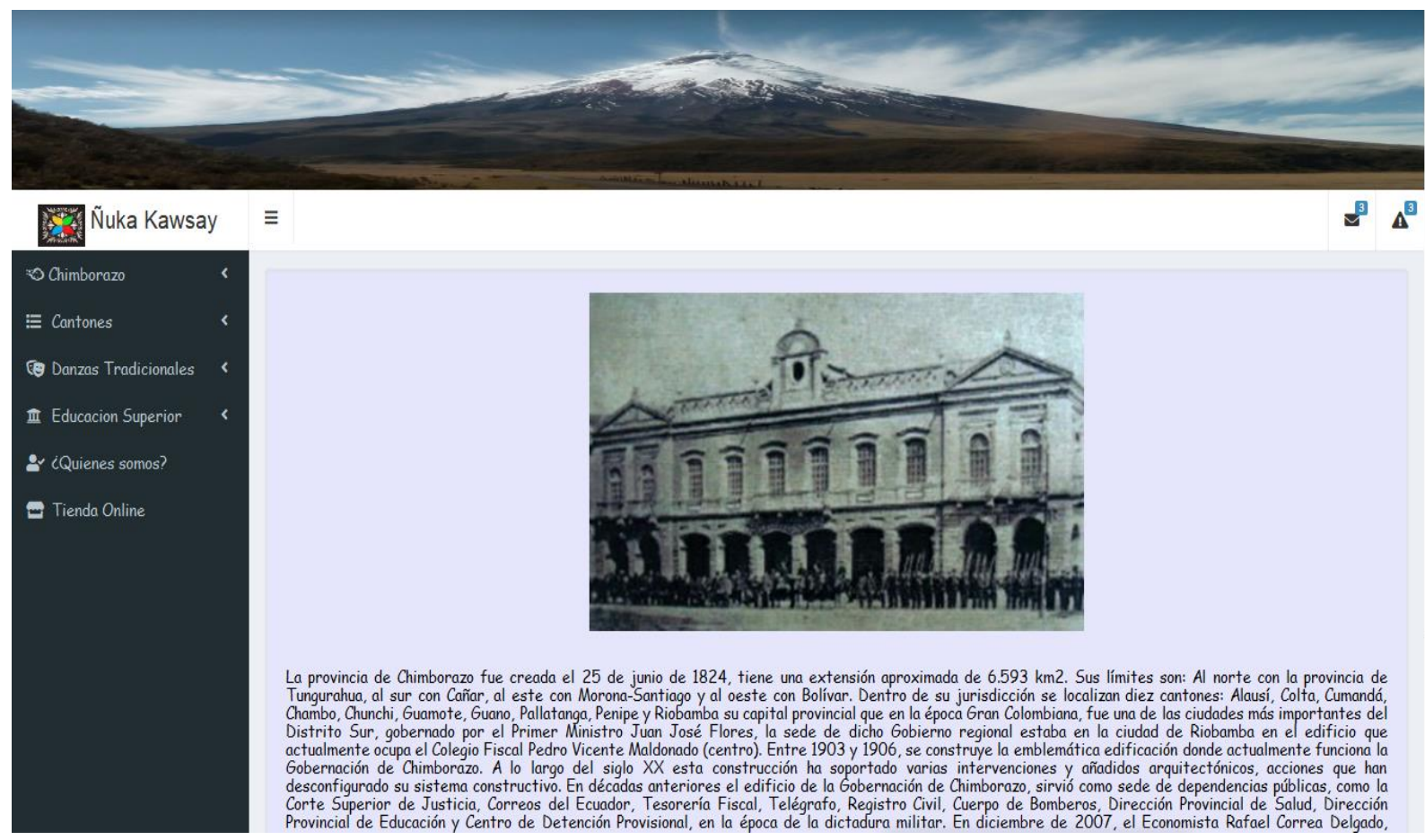

Además, la aplicación permite visualizar información sobre ubicación, población, datos estadísticos de los diferentes cantones de nuestra provincia, como se muestra en las Figuras 2, Figura 3 y Figura 4 
Figura 2. Densidad Poblacional

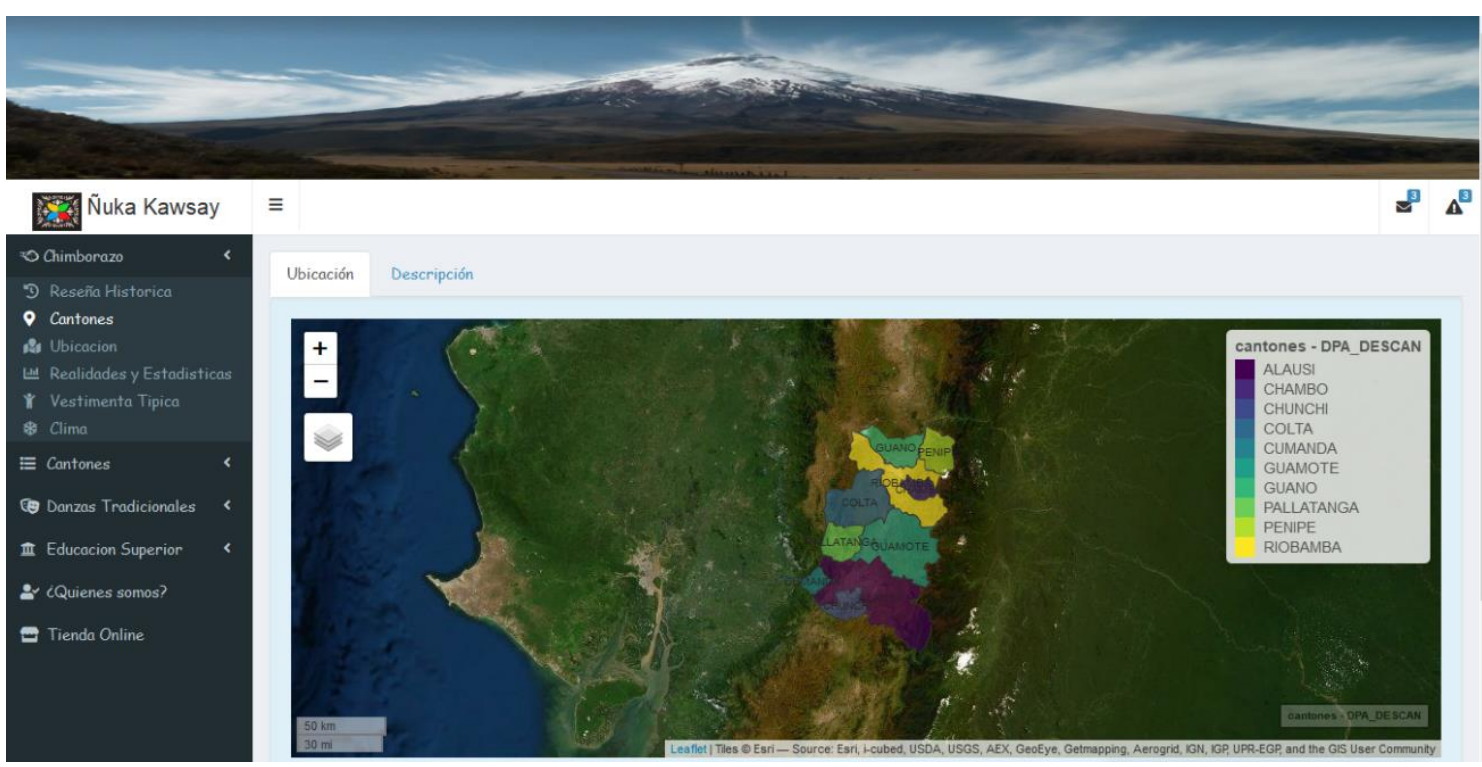

Fuente: Aplicación Informática

Elaborado por: Investigadores

Figura 3. Información estadística

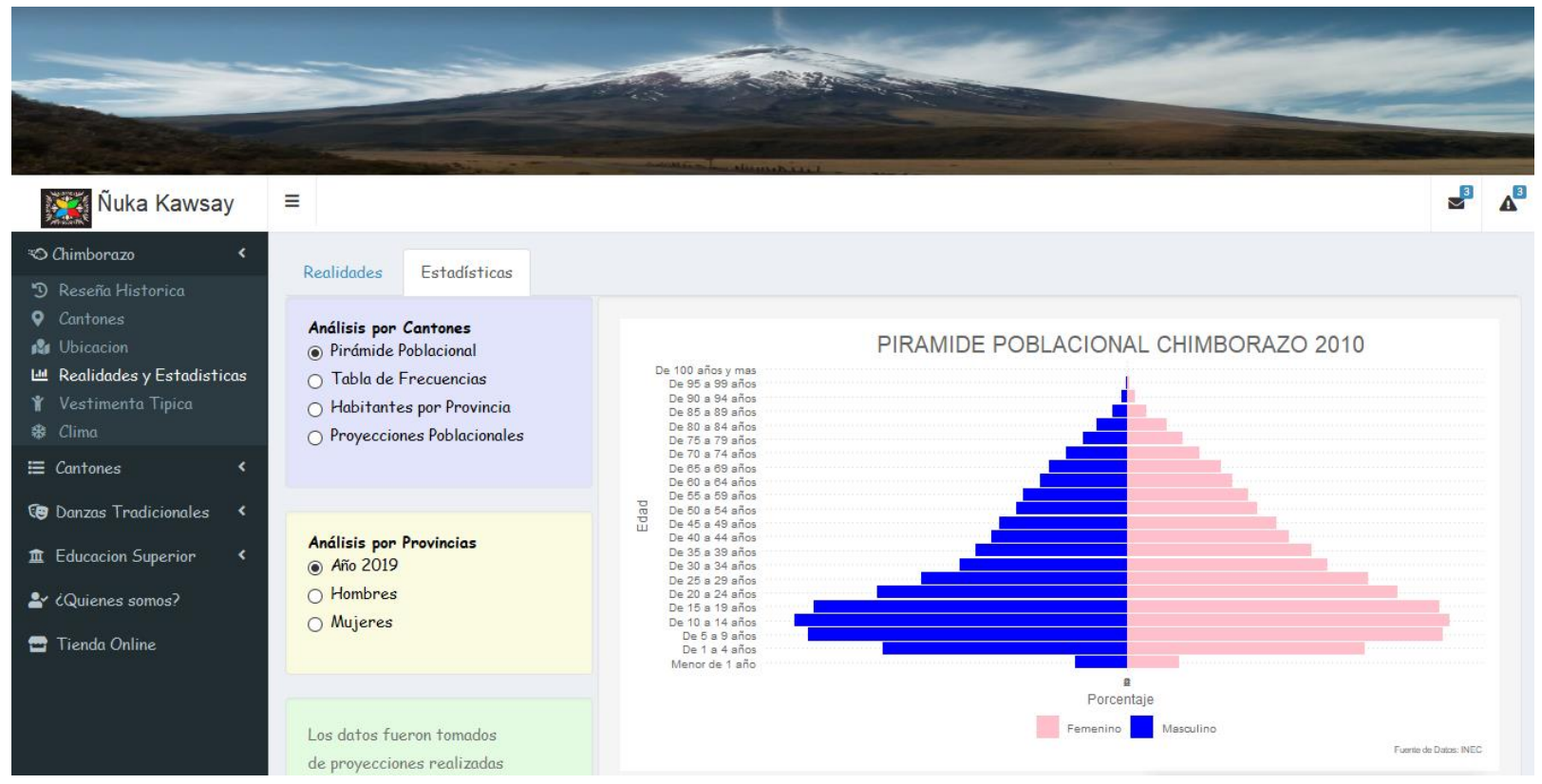

Fuente: Aplicación Informática

Elaborado por: Investigadores 
Figura 4. Información gastronómica

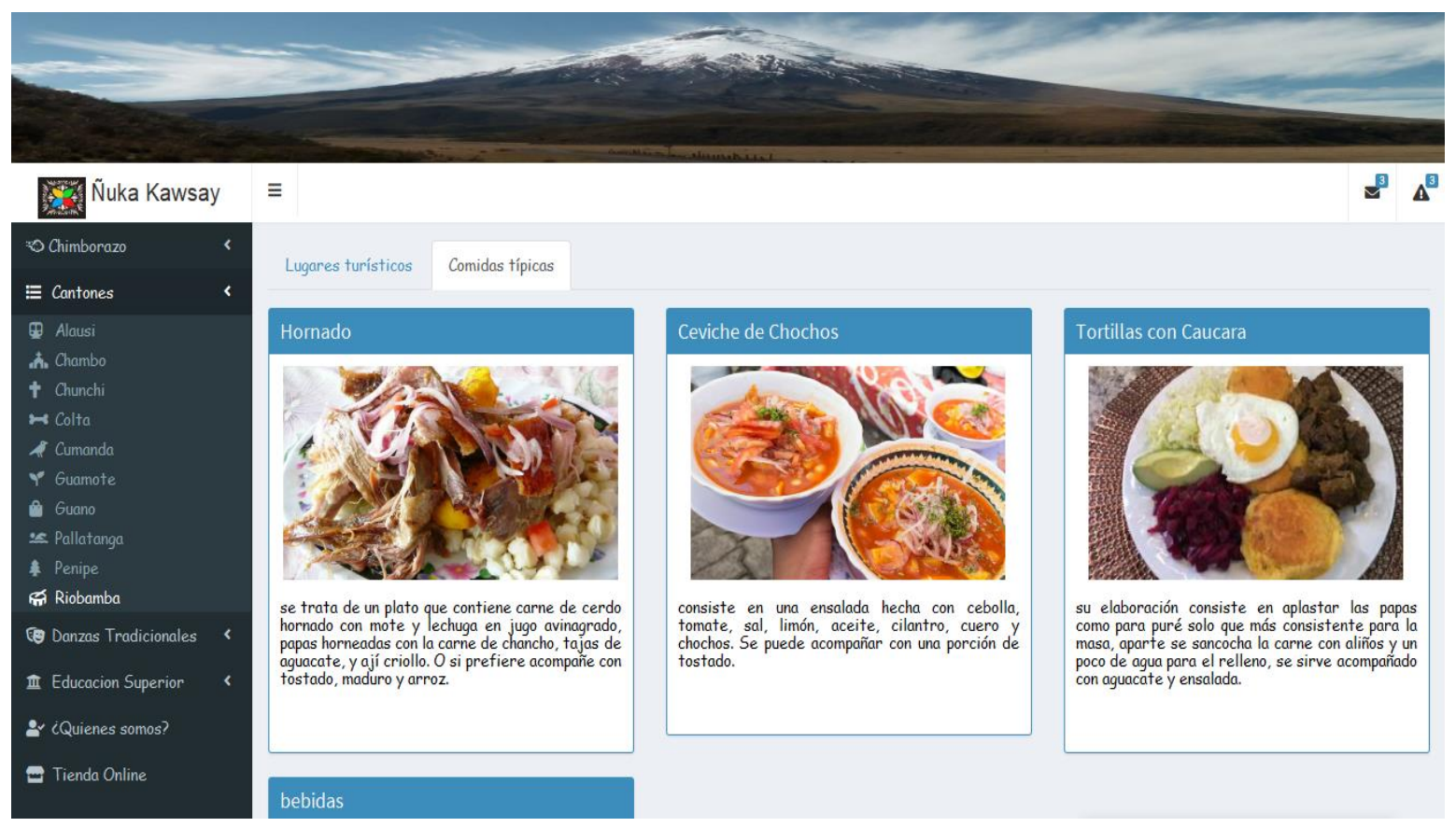

Fuente: Aplicación Informática

Elaborado por: Investigadores

\section{Población en estudio}

$$
\mathrm{n}=\frac{\mathrm{z}^{2} \mathrm{pqN}}{\mathrm{E}^{2}(\mathrm{~N}-1) \mathrm{pq}}
$$

Se consideró un muestreo probabilístico de tipo aleatorio, con el cual se determinó el tamaño de la muestra en estudio que permitió obtener la opinión de la ciudadanía, sobre la creación de la aplicación, sobre una población conocida de 458.581 habitantes, con error de 0.05 , valor de $\mathrm{Z}$ crítico de 1.96. Es decir, una muestra de 1537 habitantes quienes fueron encuestados y que permitió establecer el nivel de aceptación de la aplicación.

\section{Análisis Descriptivo}

El análisis de estadísticas descriptivas fue realizado mediante Minitab y Excel, Mediante el análisis gráfico se dio a conocer los resultados que se obtuvieron de realizar la investigación de mercados hacia la población de Chimborazo. Además, mediante funciones y fórmulas de $\mathrm{R}$, se planteó una estadística, para que el usuario interactúe en la aplicación al momento de conocer sobre ¿Cuál es la provincia con mayor o menor área?, datos obtenidos con datos de formato shapefile (.shp) mediante R, La información permitió dar forma a los mapas de ubicación de la provincia de Chimborazo y de sus cantones. 


\section{Resultados}

Sobre la muestra determinada en la Tabla 1, se realizaron encuestas sistematizadas a través de diez preguntas, que proporcionaron la información base para la investigación

Tabla 1: Pregunta 1 relacionada a ¿Cuál es su género?

\begin{tabular}{lll}
\hline Género & $\mathbf{N}^{\circ}$ encuestados & Porcentaje \\
\hline Femenino & 778 & 50,62 \\
Masculino & 759 & 49,38 \\
N & 1537 & 100
\end{tabular}

Fuente: Investigación de campo

Elaborado por: Los investigadores

Figura 5. ¿Cuál es su género?

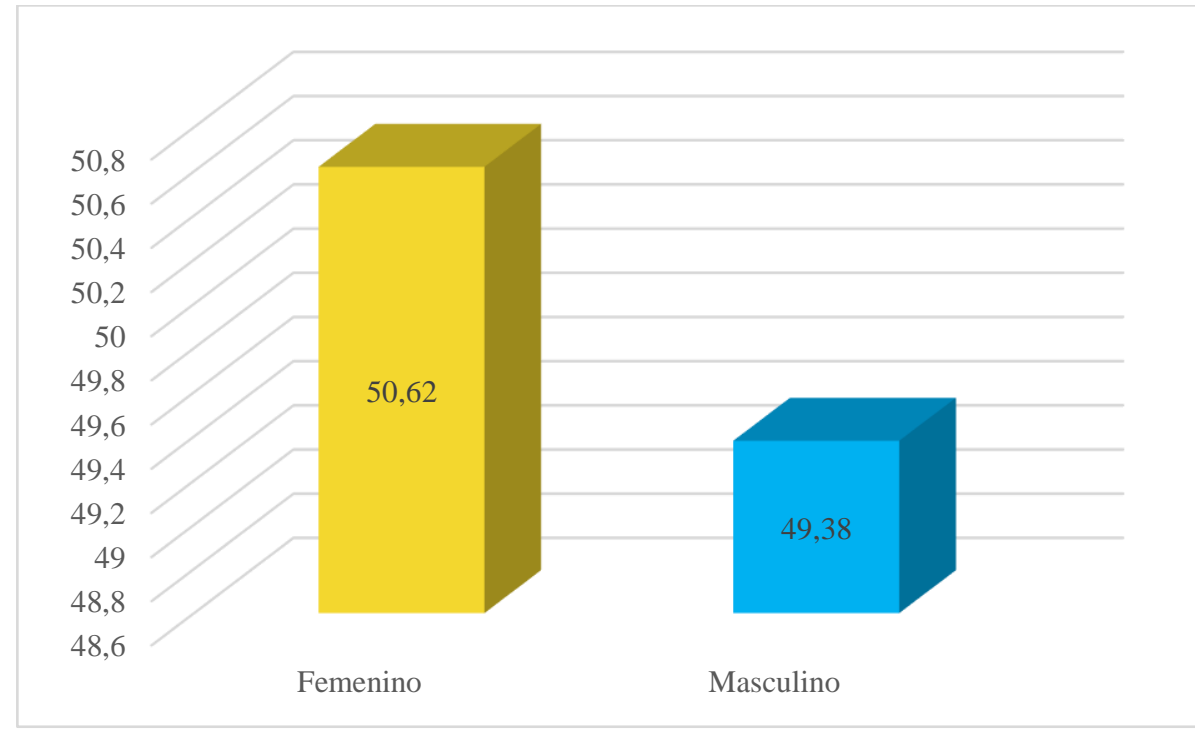

Fuente: Tabla 1

Elaborado por: Los autores

La figura 5 muestra que, de las 1537 personas encuestadas, el 50,62\% son de género femeninoy el 49,38\% de género masculino; además, dicha muestra tiene una edad promedio de 23 años con una desviación estándar de 9,49 años como se muestra en la Figura 6.

Tabla 2. Pregunta 2 relacionada a ¿Cuál es su edad?

\begin{tabular}{cccc}
\hline Media1 & EEMedia1 & Desv.Est & Varianza \\
\hline 23,0098232 & 0,24285227 & 9,48990126 & 90,0582259
\end{tabular}

Fuente: Investigación de campo

Elaborado por: Los investigadores 
Figura 6. ¿Cuál es su edad?

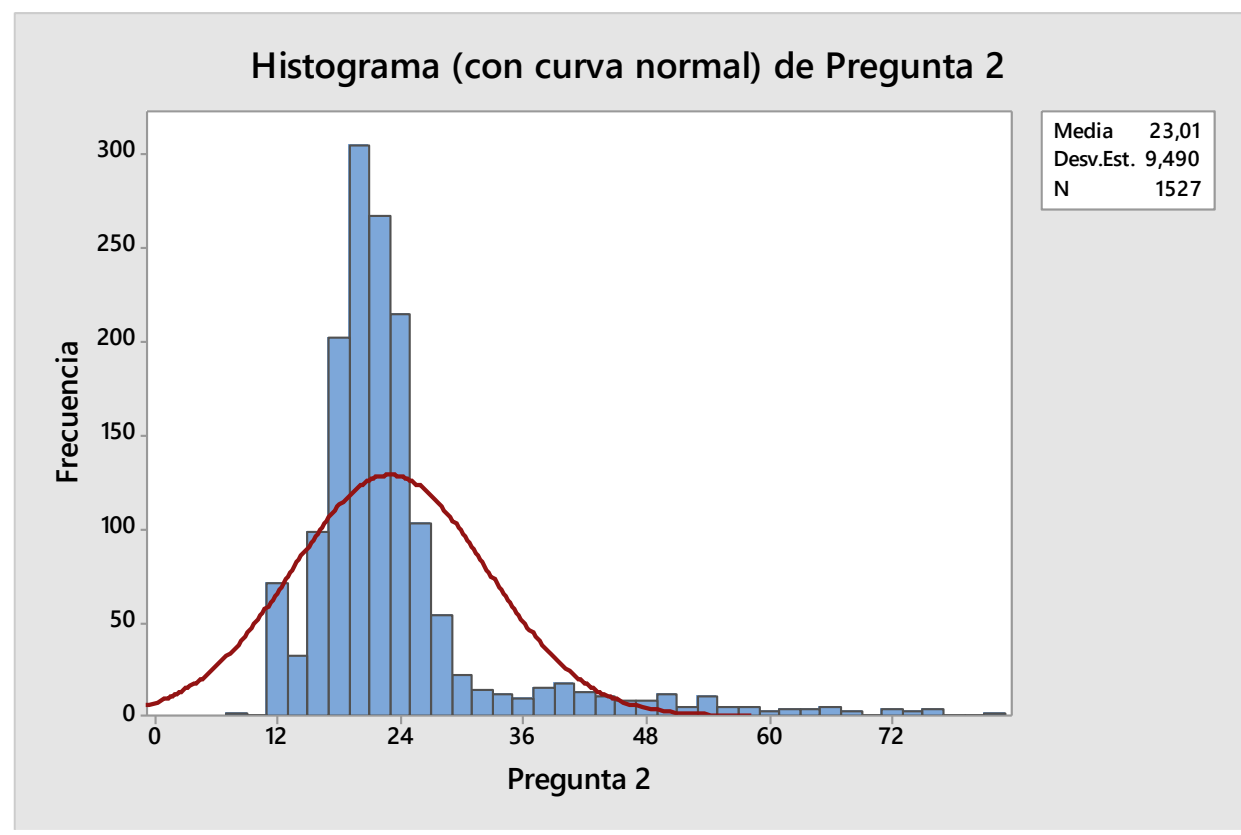

Fuente: Tabla 2

Elaborado por: Los investigadores

La tabla 3 detalla las respuestas sobre la interrogante de que, si tienen conocimiento sobre la cultura, lugares turísticos, centros de diversión entre otros, de la provincia de Chimborazo, obteniendo respuestas positivas en un 60,96\%; mientras que el valor restante que es del $38,95 \%$ no tienen conocimiento.

Tabla 3. Pregunta 3 relacionada a ¿Conoce usted sobre la cultura, lugares turísticos, centros de educación superior, gastronomía y tradiciones de la provincia de Chimborazo?

\begin{tabular}{lcc}
\hline & Conteo & Porcentaje \\
\hline No & 598 & $38,95 \%$ \\
Sí & 937 & $60,92 \%$ \\
Vacías & 2 & $0,13 \%$ \\
N & 1537 & \\
\hline
\end{tabular}

Fuente: Investigación de campo

Elaborado por: Los investigadores 
Figura 7. ¿Conoce usted sobre la cultura, lugares turísticos, centros de educación superior, gastronomía y tradiciones de la provincia de Chimborazo?

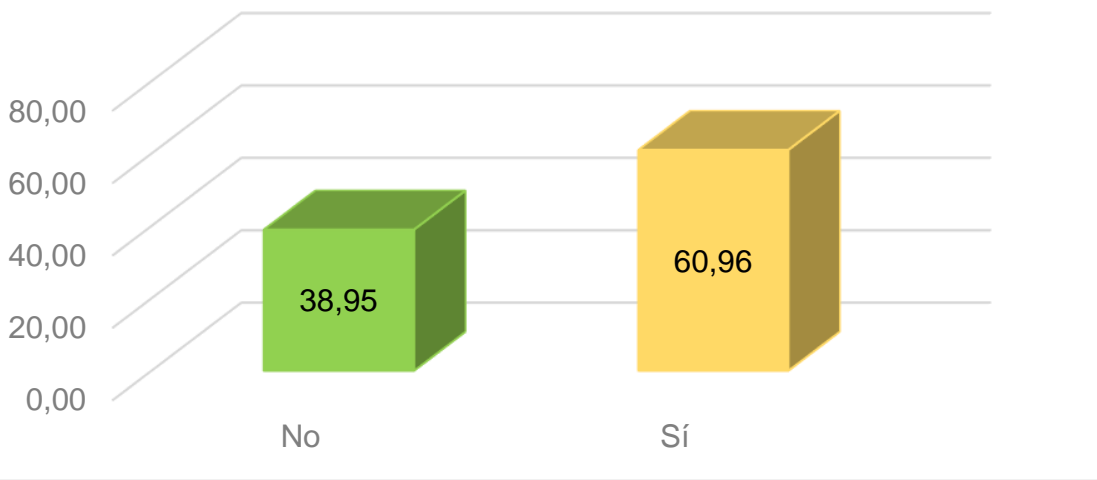

Fuente: Tabla 3

Elaborado por: Los autores

La figura 8 evidenció que, el motivo por el que las personas no conozcan sobre la cultura, lugares turísticos entre otros de la provincia de Chimborazo: se debe a que el 78,2\% manifiestan que la provincia carece de impulso turístico; mientras que el 21,8\% considera lo contrario.

Tabla 4. Pregunta 4 relacionada a ¿Considera usted que la provincia de Chimborazo carece de impulso turístico?

\begin{tabular}{lcc}
\hline & Conteo & Porcentaje \\
\hline No & 335 & $21,80 \%$ \\
Sí & 1202 & $78,20 \%$ \\
N & $\mathbf{1 5 3 7}$ & \\
\hline
\end{tabular}

Fuente: Investigación de campo

Elaborado por: Los investigadores

Figura 8. ¿Considera usted que la provincia de Chimborazo carece de impulso turístico?

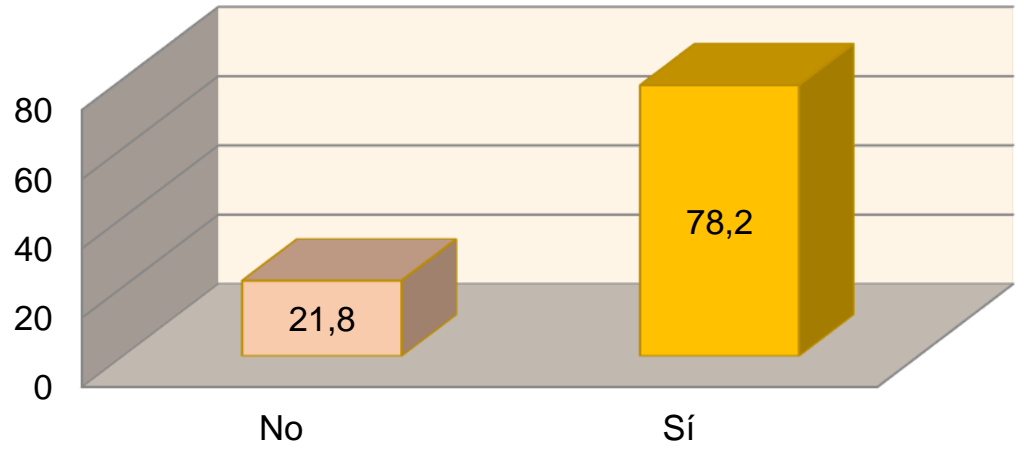

Fuente: Tabla 4

Elaborado por: Los autores 
La figura 9 evidenció que más de un $90 \%$ de los encuestados, no conocen sobre una aplicación informática que dé a conocer la cultura de la provincia de Chimborazo. Solo un $8,98 \%$ manifestaron que tenían conocimiento de alguna aplicación informática similar a la propuesta a desarrollada a través de esta investigación. Así mismo, la figura 10 evidenció el interés del 89,13\% de encuestados de disponer de la aplicación propuesta.

Tabla 5. Pregunta 5 relacionada a ¿Conoce alguna aplicación o página web que brinde información sobre gastronomía, artesanías, lugares turísticos, danzas tradicionales y educación superior acerca de la provincia de Chimborazo?

\begin{tabular}{lcc}
\hline & Conteo & Porcentaje \\
\hline No & 1398 & $90,95 \%$ \\
Sí & 138 & $8,98 \%$ \\
Vacías & 1 & $0,07 \%$ \\
N & 1537 & \\
\hline \multicolumn{3}{c}{ Fuente: Investigación de campo } \\
Elaborado por: Los autores
\end{tabular}

Figura 9. ¿Conoce alguna aplicación o página web que brinde información sobre gastronomía, artesanías, lugares turísticos, danzas tradicionales y educación superior acerca de la provincia de Chimborazo?

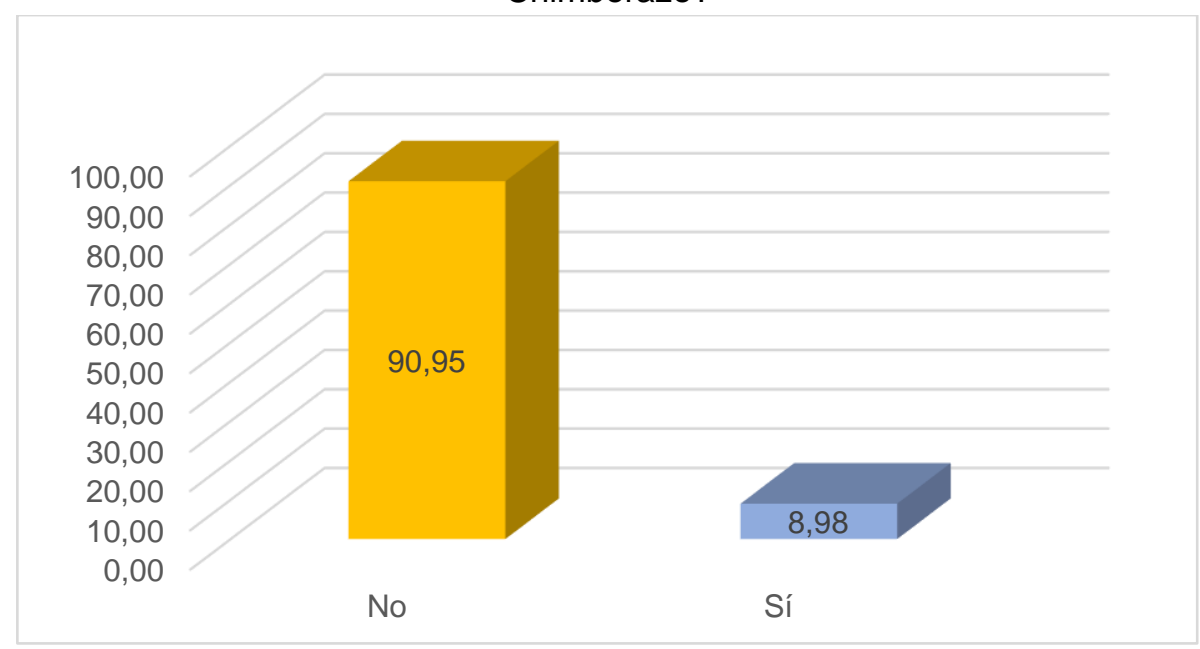

Fuente: Tabla 5

Elaborado por: Los investigadores

Tabla 6. Pregunta 6 relacionada a ¿Estaría usted interesado en una aplicación cultural mediante la cual tenga acceso a lo mencionado, en la pregunta anterior?

\begin{tabular}{lll}
\hline & Conteo & Porcentaje \\
\hline No & 167 & $10,87 \%$ \\
Sí & 1370 & $89,13 \%$ \\
N & 1537 & \\
\hline
\end{tabular}

Fuente: Investigación de campo

Elaborado por: Los investigadores 
Figura 10. ¿Estaría usted interesado en una aplicación cultural mediante la cual tenga acceso a lo mencionado, en la pregunta anterior?

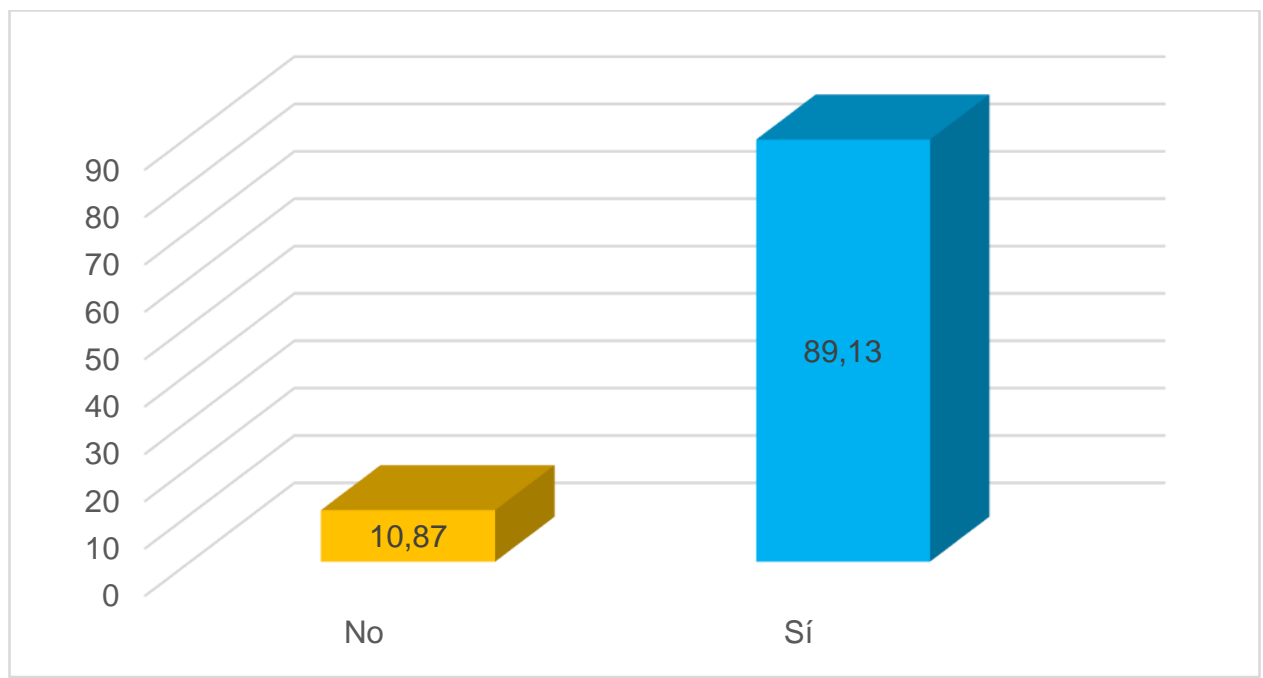

Fuente: Tabla 6

Elaborado por: Los investigadores

La tabla 7, muestra el detalle del tipo de información que llamaría más la atención de la aplicación cultural, obteniéndose que el $89,13 \%$ de las personas encuestadas están interesados en la aplicación; mientras que el $10,87 \%$ no está interesado con respecto al contenido que ofertará la aplicación. El 29.69\% de las personas están interesados en conocer toda la información de la página (Educación, Artesanías, Danzas Tradicionales, Gastronomía y Turismo). Se recalca que existe mayor interés en la sección turística con una aceptación del $25,88 \%$.

Tabla 7. Pregunta 7 relacionada a ¿Qué información le llamaría más la atención de la aplicación cultural?

\begin{tabular}{lll}
\hline & Conteo & Porcentaje \\
\hline Otras & 7 & $0,37 \%$ \\
Sección Danzas Tradicionales & 182 & $9,61 \%$ \\
Sección de artesanías (Tienda & 180 & $9,51 \%$ \\
Online) & & \\
Sección educativa & 115 & $6,08 \%$ \\
Sección gastronómica & 357 & $18,86 \%$ \\
Sección turística & 490 & $25,88 \%$ \\
Todas las anteriores & 562 & $29,69 \%$ \\
$\mathbf{N}$ & $\mathbf{1 8 9 3}$ & $\mathbf{1 0 0 , 0 0 \%}$ \\
\hline
\end{tabular}

Fuente: Investigación de campo Elaborado por: Los investigadores 
Figura 11. ¿Qué información le llamaría más la atención de la aplicación cultural?

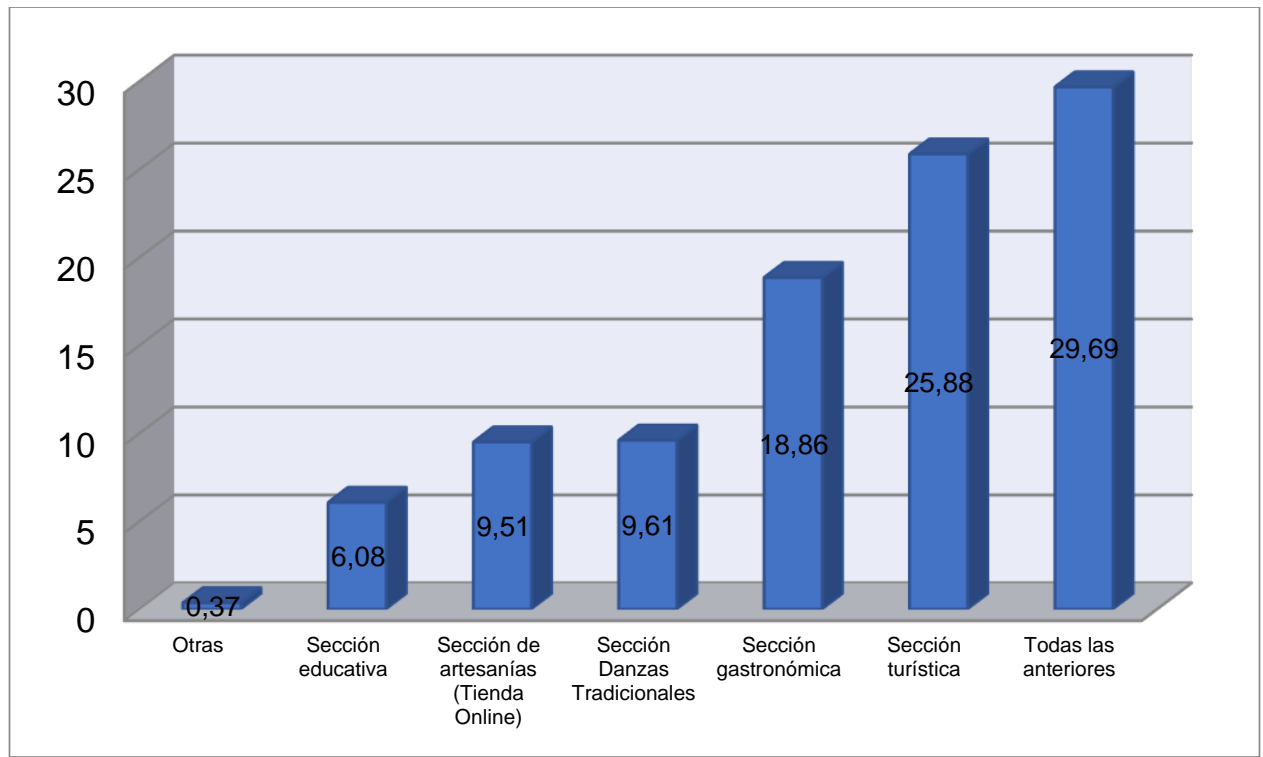

Fuente: Tabla 7

Elaborado por: Los autores

La tabla 8, describe los resultados referentes a los medios a través de los cuales les gustaría a los encuestados recibir publicidad e información de la aplicación informática, obteniendo que al 73,35\% de las personas les gustaría recibir la publicidad mediante las redes sociales, seguido por un $17,69 \%$ de personas que les gustaría conocer la aplicación mediante publicidad televisiva.

Tabla 8. Pregunta 8 relacionada a ¿A través de qué medios le gustaría recibir publicidad e información de la aplicación?

\begin{tabular}{lll}
\hline & Conteo & Porcentaje \\
\hline Afiches Informativos & 93 & $5,52 \%$ \\
Otros & 5 & $0,30 \%$ \\
Portal de la Gobernación de & 53 & $3,15 \%$ \\
Chimborazo & & \\
Redes Sociales & 1236 & $73,35 \%$ \\
Televisión & 298 & $17,69 \%$ \\
N & 1685 & $100,00 \%$ \\
\hline
\end{tabular}

Fuente: Investigación de campo

Elaborado por: Los investigadores 
Figura 12. ¿A través de qué medios le gustaría recibir publicidad e información de la aplicación?

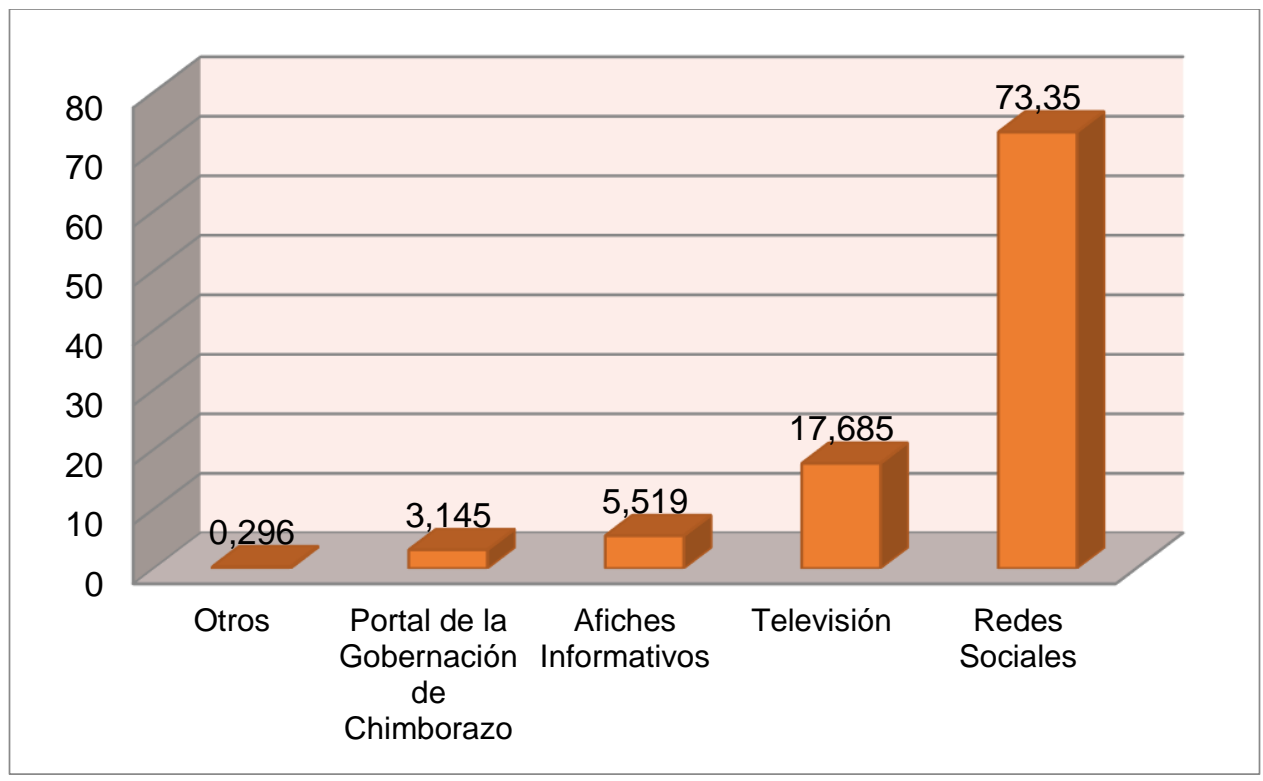

Fuente: Tabla 8

Elaborado por: Los investigadores

En la tabla 9, se muestra los resultados obtenidos referentes a los costos de las artesanías que estarían dispuestos a pagar los encuestados utilizando la tienda online implementada en la Aplicación Informática, obteniéndose que el $51,14 \%$ de los usuarios estarían dispuestos a gastar un valor menor a $\$ 10,00$, seguido por un $43,85 \%$ que gastarían entre $\$ 10,00$ y $\$ 20,00$ en cuanto a artesanías

Tabla 9. Pregunta 9 relacionada a: A la hora de comprar las artesanías que se ofertarán en la aplicación ¿Cuánto estaría dispuesto a gastar?

\begin{tabular}{lll}
\hline & Conteo & Porcentaje \\
\hline Menor a \$10 & 786 & $51,14 \%$ \\
Entre $\$ 10-\$ 20$ & 674 & $43,85 \%$ \\
Más de \$20 & 73 & $4,75 \%$ \\
Vacias & 4 & $0,26 \%$ \\
$\mathrm{~N}$ & 1537 & $100 \%$ \\
\hline
\end{tabular}

Fuente: Investigación de campo

Elaborado por: Los investigadores 
Figura 13. A la hora de comprar las artesanías que se ofertarán en la aplicación ¿Cuánto estaría dispuesto a gastar?

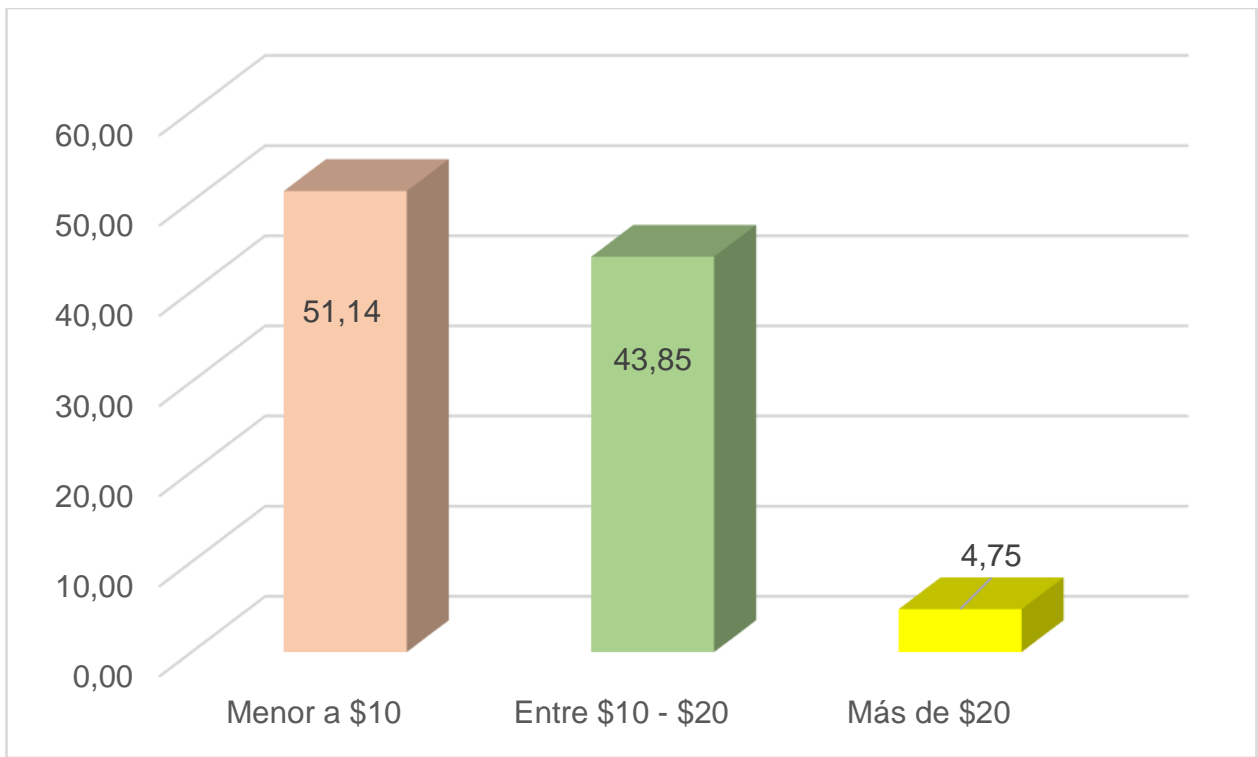

Fuente: Tabla 9

Elaborado por: Los investigadores

La pregunta 10, permitió identificar funcionalidades adicionales en la aplicación informática como: lugares con infraestructura deportiva, biografías de personajes relevantes en cada uno de los cantones.

Los resultados obtenidos a través de las encuestas realizadas, permitieron justificar el desarrollo de la aplicación informática cultural - educativa elaborada en Shiny, notándose que la muestra estudiada, considera que la provincia de Chimborazo carece de impulso turístico por la falta de motivación y atención a este sector por parte del GAD de la provincia de Chimborazo, lo cual da resultados positivos para el presente estudio puesto que se podrá con esto incentivar la parte tecnológica y estadística en la cual se está enfocando al crear la aplicación informática cultural.

En la actualidad existen páginas como GoRaymi y VisitaEcuador, que expone de forma generalizada las diversas culturas, tradiciones y lugares turísticos de cada cantón del Ecuador. Por esta razón, la implementación de la aplicación web interactiva "Sumak Kawsay" utilizando R-Shiny, se justifica y se valida a través de las encuestas realizadas. Esta plataforma tecnológica permitirá a largo plazo, que pequeñas empresas que elaboran productos y servicios autóctonos de la provincia tengan mayor oportunidad de desarrollo cultural, social y económico. 


\section{Conclusiones}

- Se concluye que la principal temática cultural a ser promocionada dentro de la provincia de Chimborazo es la "sección de turismo" con un porcentaje de aceptación del 25,88\% y la "sección gastronómica" con un 18,86\%; permitiendo así, que dicha información sea implementada en la aplicación informativa.

- A través de la promoción online mediante la plataforma tecnológica con R Shiny de sitios turísticos de la provincia de Chimborazo, se puede generar información estadística muy crucial para la toma de decisiones, respecto a la actualización continua del portafolio de atractivos turísticos de los diez cantones en base a la oferta y la demanda que se genere en el tiempo.

- Con la información obtenida de las encuestas aplicadas, se pudo evidenciar que la aplicación informativa cultural Ñuka Kawsay, presentó un gran porcentaje de aceptación por parte de la población de la provincia de Chimborazo, debido a que existe un gran desconocimiento de la información turística, cultural de la misma; permitiendo de esta manera dar a conocer la Provincia y aportar al crecimiento económico.

- El desarrollo de dicha aplicación, incide de manera positiva a las entidades gubernamentales como el ministerio de turismo e indirectamente a los Gobiernos Autonomos Desentralizados GAD de cada cantón de la provincia de Chimborazo, que pueden complementar las páginas de los municipios con la aplicación y además a determinados agentes económicos interesados en la aplicación; como son las agencias de viajes, establecimientos de comidas típicas, grupos de danzas tradicionales y en general todos aquellos que deseen publicitar sus productos y servicios autóctonos de la provincia de Chimborazo. También ofrece la posibilidad de iniciar nuevos estudios como determinar la satisfacción de clientes que usen la aplicación implementada en la investigación, sin olvidarnos que dicha aplicación beneficiará en el ámbito educativo a las 2 entidades renombradas a nivel de la provincia de Chimborazo como son La Escuela Superior Politécnica de Chimborazo y la Universidad Nacional de Chimborazo.

\section{Referencias Bibliográicas}

Pizam, A., Neumann, Y., \& Reichel A. (1978). Dimensions of tourist satisfaction with a destination area. Annals of Tourism Research, 5, 314-322.

Leon, B. (2018). Paisaje cultural y una nueva forma de entender el Patrimonio en

Ecuador. Rev. interam. ambient. tur. [online]. 2018, vol.14, n.2 [citado 2019-05-28], pp.161-169. Disponible en:

$<$ https://scielo.conicyt.cl/scielo.php?script=sci_arttext\&pid=S0718-

235X2018000200161\&lng=es\&nrm=iso>. ISSN 0717-6651. http://dx.doi.org/10.4067/S0718-235X2018000200161.

Martín, J., \& Bustamante-Sánchez N. (2019). A cultural analysis of the secondary housing tourism in Vilcabamba, Ecuador. International Journal of Housing Markets and Analysis, IJHMA-05-2018-0028. https://doi.org/10.1108/IJHMA-05-2018-0028 
Cànoves, G., Herrera L., \& Cuesta L. (2005). Turismo rural en Cataluña: condicionantes de la oferta y la demanda. Investigaciones Geográficas (Esp), (37), 5-26.

Gómez, D., Molina, M., Mulero, J., Nueda, M., \& Pascual, R. (2016). Aplicaciones diseñadas con Shiny: un recurso docente para la enseñanza de la estadística. Retrieved from http://rua.ua.es/dspace/handle/10045/59411

Crosby, A. (1996). Elementos básicos para un turismo sostenible en las áreas naturales. Forum Natura.

Gurría, M. (2004). Introducción al Turismo. Editorial Trillas. México.

Matute y Asanza. (2006). Aspectos sociopsicológicos del turismo. Consultado el 14 de noviembre del 2006, en http://www. eumed.net/libros/2006c/209/index.htm

Ramírez, R. (2006). Definición del Turismo. Venezuela, conciencia, turismo y desarrollo. Consultado el 03 de noviembre del 2006 en http://www.venezuelatuya.com/articulos/turismo0010.htm. 


\section{PARA CITAR EL ARTÍCULO INDEXADO.}

Paredes Castelo, L., Pilco Ninabanda, D., Manzo Moreira, B. del C., \& Rodríguez Mayorga, M. (2019). Implementación de una aplicación informativa cultural - educativa mediante Shiny para la provincia $\begin{array}{llll}\text { de Chimborazo. Ciencia 3igital, 3.2.1), 370. } & \text { 354-370 }\end{array}$ https://doi.org/10.33262/cienciadigital.v3i3.2.1.891

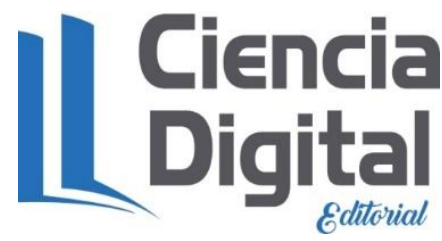

El artículo que se publica es de exclusiva responsabilidad de los autores y no necesariamente reflejan el pensamiento de la Revista Ciencia Digital.

El artículo queda en propiedad de la revista y, por tanto, su publicación parcial y/o total en otro medio tiene que ser autorizado por el director de la Revista Ciencia Digital.
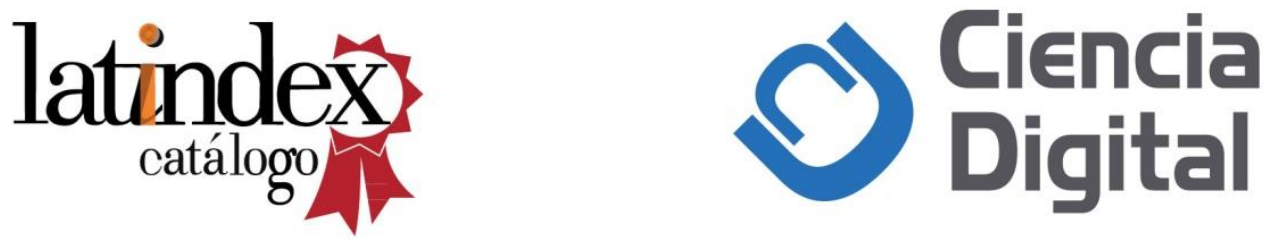